Article

\title{
Pharmacogenetics of OATP Transporters Reveals That SLCO1B1 c.388A $>$ G Variant Is Determinant of Increased Atorvastatin Response
}

Alice C. Rodrigues ${ }^{1, *}$, Paula M. S. Perin ${ }^{1}$, Sheila G. Purim ${ }^{2}$, Vivian N. Silbiger ${ }^{1}$, Fabiana D. V. Genvigir ${ }^{1}$, Maria Alice V. Willrich ${ }^{1}$, Simone S. Arazi ${ }^{1}$, Andre D. Luchessi ${ }^{1}$, Mario H. Hirata ${ }^{1}$, Marcia M. S. Bernik ${ }^{3}$, Egidio L. Dorea ${ }^{3}$, Carla Santos ${ }^{4,5}$, Andre A. Faludi ${ }^{6}$, Marcelo C. Bertolami ${ }^{6}$, Antonio Salas ${ }^{5}$, Ana Freire ${ }^{5}$, Maria V. Lareu ${ }^{5}$, Christopher Phillips ${ }^{5}$, Liliana Porras-Hurtado ${ }^{5}$, Manuel Fondevila ${ }^{5}$, Angel Carracedo ${ }^{5}$ and Rosario D. C. Hirata ${ }^{1}$

1 Faculty of Pharmaceutical Sciences, University of Sao Paulo, Sao Paulo 05508-900, Brazil; E-Mails: paulamsp@gmail.com (P.M.S.P.); viviansilbiger@hotmail.com (V.N.S.); fdallavecchia@yahoo.com.br (F.D.V.G.); malicewi@usp.br (M.A.V.W.); sisorkin@usp.br (S.S.A.); adluchessi@uol.com.br (A.D.L.); mhhirata@usp.br (M.H.H.); rosariohirata@usp.br (R.D.C.H.)

2 Life Technologies, Sao Paulo 04311-000, Brazil; E-Mail: sheila.purim@lifetech.com

3 University Hospital, University of Sao Paulo, Sao Paulo 05508-000, Brazil;

E-Mails: mbernik@usp.br (M.M.S.B.); egidiodr@gmail.com (E.L.D.)

4 Department of Biology, University of Aveiro, Aveiro 3810-193, Portugal;

E-Mail: carla.santo@ispa.pt

5 Forensic Genetics Unit, Institute of Legal Medicine, University of Santiago de Compostela, Galicia 15705, Spain; E-Mails: antonio.salas@usc.es (A.S.); ana.freire@usc.es (A.F.);

mvictoria.lareu@usc.es (M.V.L.); christopherpaul.phillips@usc.es (C.P.); glolipo@gmail.com (L.P.-H.); manuel.fondevila@usc.es (M.F.); apimlang@usc.es (A.C.)

6 Dante Pazzanese Institute, Sao Paulo 04012-909, Brazil; E-Mail: afaludi@uol.com.br (A.A.F.); bertolami@uol.com.br (M.C.B.)

* Author to whom correspondence should be addressed; E-Mail: alice-rodrigues@usp.br; Tel.: +55-11-3091-3660; Fax: +55-11-3813-2197.

Received: 29 July 2011; in revised form: 29 August 2011 / Accepted: 30 August 2011 / Published: 9 September 2011

Abstract: Aims: The relationship between variants in SLCO1B1 and SLCO2B1 genes and lipid-lowering response to atorvastatin was investigated. Material and Methods: One-hundred-thirty-six unrelated individuals with hypercholesterolemia were selected and 
treated with atorvastatin $(10 \mathrm{mg} /$ day/4 weeks $)$. They were genotyped with a panel of ancestry informative markers for individual African component of ancestry (ACA) estimation by SNaPshot ${ }^{\circledR}$ and SLCO1B1 (c.388A $>$ G, c.463C $>$ A and c.521T $>$ C) and SLCO2B1 $(-71 \mathrm{~T}>\mathrm{C})$ gene polymorphisms were identified by TaqMan ${ }^{\circledR}$ Real-time PCR. Results: Subjects carrying SLCO1B1 c.388GG genotype exhibited significantly high low-density lipoprotein (LDL) cholesterol reduction relative to c.388AA+c.388AG carriers (41 vs. $37 \%, p=0.034$ ). Haplotype analysis revealed that homozygous of SLCO1B1*15 (c.521C and c.388G) variant had similar response to statin relative to heterozygous and non-carriers. A multivariate logistic regression analysis confirmed that c.388GG genotype was associated with higher LDL cholesterol reduction in the study population (OR: 3.2, CI95\%:1.3-8.0, $p<0.05$ ). Conclusion: SLCO1B1 c.388A $>\mathrm{G}$ polymorphism causes significant increase in atorvastatin response and may be an important marker for predicting efficacy of lipid-lowering therapy.

Keywords: OATP; atorvastatin; single nucleotide polymorphisms; pharmacogenetics

\section{Introduction}

Organic anion transporting polypeptides (OATPs) are plasma membrane transport proteins that mediate the active cellular influx of a variety of amphipathic compounds. OATP1B1, OATP2B1 and OATP1B3 are expressed in the sinusoidal membrane of hepatocytes and transport a large number of therapeutic drugs, such as statins [1]. The uptake of statins by OATPs not only represents the first step of hepatic drug elimination, but is also a delivery system to the liver as the target organ. Such transport therefore potentially influences the efficacy of the therapy of this drug class, as differences in OATP activity may result in variability of statins plasma levels and consequently variability in drug response.

Atorvastatin is a potent inhibitor of the 3-hydroxy-3-methlyglutaryl-coenzyme A reductase (HMGCR), the rate-limiting enzyme in the cholesterol biosynthesis pathway [2]. It plays an important role in reducing plasma low-density lipoprotein (LDL) cholesterol and in preventing the risk of coronary artery disease (CAD) [3,4]. Hepatic uptake of atorvastatin has been demonstrated to be mediated in an OATP-dependent manner [5].

OATP1B1 and 2B1 are codified by solute carrier organic anion transporter family genes, member 1B1 (SLCO1B1) and 2B1 (SLCO2B1), respectively. SLCO1B1 has several common polymorphisms and its relation with statin efficacy remains uncertain. The single nucleotide polymorphism (SNP) SLCO1B1 c.521T $>\mathrm{C}$ has been associated with markedly increased plasma concentrations of simvastatin, rosuvastatin, pravastatin, and atorvastatin [6-12]. These studies have shown that homozygous for c.521C allele presented the highest plasma concentration as compared to TC heterozygote or TT homozygote. The increase in plasma concentration of statins may increase the exposure of the drug and lead to adverse drug reactions. Indeed, SLCO1B1*5 (c.521C) was associated with increased risk of statin-induced myopathy in a genome-wide association study in patients taking simvastatin $80 \mathrm{mg}$ [13]. The SNP c.388A $>\mathrm{G}\left(S L C O 1 B 1^{*} 1 b\right)$ has been also associated with higher activity of OATP1B1 resulting in lower oral bioavailability of pravastatin [14]. 
Many studies have focused only on the pharmacokinetics of statin, whereas the impact of SLCO1B1 genotypes on lipid-lowering response to statins remains unsure. In one study, in Japanese hypercholesterolemic patients treated with pravastatin for eight weeks, heterozygous carriers of SLCO1B1*15 allele (c.388G and c.521C alleles) had poor LDL cholesterol reduction as compared with non-carriers (reduction: -14.1 vs. $-28.9 \%$ ) [15]. On the other hand, in a cohort of elderly hypercholesterolemic patients treated with fluvastatin extended-release, the SLCO1B1 c.463C $>$ A SNP was significantly associated with enhanced fluvastatin response [16].

The potential contribution of genetic variations in $S L C O 2 B 1$ in statins efficacy is not known. Until now, only one study has accessed the impact of variants of $S L C O 2 B 1$; however, no differences were found [7]. OATP2B1, differently from OATP1B1 is localized not only in hepatocytes, but at membranes of enterocytes, human skeletal muscle and heart. Recent studies have suggested that OATP2B1 may play a role in statin-induced myopathy, since the presence of OATP2B1 in primary muscle myoblast cells caused a significant increase in intracellular retention of statins [17].

The uptake and delivery of atorvastatin into hepatocytes by OATP is essential for its action. Because some studies have previously associated OATP variants with altered pharmacokinetic profile of atorvastatin, the aim of this study was to describe the influence of SLCO1B1 and SLCO2B1 genotypes on the pharmacological efficacy of atorvastatin.

\section{Results and Discussion}

\subsection{Characteristics of the Hypercholesterolemic Individuals}

Clinical and laboratory data of the $\mathrm{HC}$ subjects were previously described by Rebecchi et al. (2009) [18]. Atorvastatin treatment significantly reduced total LDL cholesterol and triglycerides values (Table 1). Concomitant ingestion of CYP3A4 substrates or inhibitors did not affect atorvastatin response ( $p>0.05)$, as evaluated by Chi-square test (data not shown). We did not observe an increase in high-density lipoprotein (HDL) cholesterol levels as it has been described for this drug. In addition, atorvastatin treatment did not cause a significant increase in CK levels. There was no report of intolerance or adverse effects related to atorvastatin therapy. We have observed an increase of ALT levels after treatment, but this increase did not translate into hepatotoxicity for the patients that have undergone atorvastatin treatment.

Table 1. Biochemical profile of hypercholesterolemic individuals in response to atorvastatin (10 mg/day/4 weeks).

\begin{tabular}{lcccc}
\hline Variables & Basal & Atorvastatin * & Change (\%) & $\boldsymbol{P}$ \\
\hline TC $(\mathrm{mg} / \mathrm{dL})$ & $281 \pm 38$ & $198 \pm 30$ & $-28.9 \pm 9.5$ & $<0.001$ \\
LDL-C (mg/dL) & $193 \pm 55$ & $118 \pm 27$ & $-38.3 \pm 12.4$ & $<0.001$ \\
HDL-C (mg/dL) & $56 \pm 14$ & $54 \pm 13$ & $-2.5 \pm 10.5$ & $<0.002$ \\
TG (mg/dL) & $160 \pm 66$ & $132 \pm 52$ & $-26.9 \pm 52.5$ & $<0.001$ \\
CK (U/L) & $102 \pm 80$ & $104 \pm 88$ & $4.9 \pm 36.5$ & 0.606 \\
ALT (U/L) & $22 \pm 10$ & $25 \pm 15$ & $23.0 \pm 63.2$ & $<0.001$ \\
ApoAI (g/L) & $130 \pm 25$ & $136 \pm 27$ & $4.9 \pm 15.4$ & 0.013 \\
ApoB (g/L) & $140 \pm 22$ & $102 \pm 22$ & $-28 \pm 46.1$ & $<0.001$ \\
\hline
\end{tabular}

TC: total cholesterol, LDL-C: low-density lipoprotein cholesterol; HDL-C: high-density lipoprotein cholesterol; TG: triglyceride; CK: Creatine kinase; ALT: Alanine aminotransferase; ApoAI: Apolipoprotein AI; ApoB: Apolipoprotein B. * 10mg/daily for 4 weeks. 


\subsection{SLCO1B1 and SLCO2B1 Polymorphisms}

Genotype and allele frequencies for $S L C O 1 B 1$ and $S L C O 2 B 1$ polymorphisms were calculated for this sample of the Brazilian population. As expected, allele frequencies of these variants were in Hardy-Weinberg Equilibrium confirming the random selection of the individuals. The frequencies of the three variants (c.388A $>$ G, c. $463 \mathrm{C}>\mathrm{A}$ and c.521T $>\mathrm{C}$ ) for $S L C O 1 B 1$ gene in Brazilian individuals were $32 \%, 16 \%$ and $12 \%$, respectively. Minor allele frequency for SLCO2B1-71C allele was 53\%.

Linkage disequilibrium was tested for SLCO1B1 variants. Association was found between c. $388 \mathrm{~A}>\mathrm{G}$ and c.521T $>\mathrm{C}$ polymorphisms $\left(D^{\prime}=0.84 ; \chi^{2}=9.56, p=0.049\right)$ and c.388A $>\mathrm{G}$ and c. $463 \mathrm{C}>\mathrm{A}$ SNPs were also consistently associated $\left(D^{\prime}=1.0 ; \chi^{2}=69.94, p<0.0001\right)$. Nevertheless, c.521T $>\mathrm{C}$ and c.463C $>\mathrm{A}$ were not associated $\left(\chi^{2}=2.32, p=0.677\right)$. Therefore, six SLCO1B1 haplotypes were found in our study group: *1a $(39.3 \%), * 1 \mathrm{~b}(33.3 \%), * 14(16.0 \%), * 15(10.3 \%)$, and $* 4(1.1 \%)$.

The frequency of SLCO1B1 and SLCO2B1 SNPs and of their haplotypes varies largely among ethnically identified populations [19-21]. Despite the fact that the described frequencies above for SLCO1B1 are similar to others previously reported [16,20], Brazilians are a highly admixed population with Amerindian, European and African ancestral roots and estimation of the genetic ancestry provided by AIMs may allow more realistic representations of such diversity [22-25]. For this purpose, we have estimated the ACA mean value for our sample and associated it with the alleles of SLC polymorphisms.

The individual ACA values across the study population ranged from 0.003 to 0.989 . ACA mean values between ancestral and variant allele of each SNP are presented in Figure S1. We observed that, only for $S L C O 2 B 1-71 \mathrm{~T}>\mathrm{C}$ polymorphism, the ACA mean value was significantly higher in subjects carrying $-71 \mathrm{~T}$ allele compared to $-71 \mathrm{C}$ allele carriers $[0.461(0.010-0.687)$ vs. $0.112(0.037-0.243)$, $p=0.023]$.

Categorization of ACA values in four quartiles $(<0.25 ; 0.25-0.50 ; 0.50-0.75 ;>0.75)$ revealed that frequency of the $S L C O 2 B 1-71 \mathrm{C}$ allele decreased progressively from the lowest $(<0.25 \mathrm{ACA})$ to the highest $(>0.75$ ACA) quartile, showing its higher prevalence in people with minor African influence (Supplemental Table 1). For SLCO1B1 gene, the frequencies of the SNPs were not different among the ACA quartiles.

SLCO1B1 c.463C $>$ A SNP showed a trend for decreasing the frequency of c.463A variant from low ACA values $(<25 \%)$ to high ACA values $(<75 \%)$ (Supplemental Figure 1, Supplemental Table 1).

These results are in agreement with previous reports showing a low prevalence of this allele in African Americans and a high prevalence in Caucasians [20]. For SLCO2B1 variant, a significant association between $-71 \mathrm{C}$ allele and ACA values was found. There is no study reporting this relationship, but we may conclude that $-71 \mathrm{C}$ allele varies among ethically identified populations and presents a low frequency in people with high African background.

The variables' age, BMI, gender, hypertension, obesity, menopause, cigarette smoking, alcohol consumption, physical activity, and baseline mean plasma lipid parameters were not different among the genotypes or haplotypes for all the polymorphisms studied (data not shown). These results suggest that $S L C O 1 B 1$ and $S L C O 2 B 1$ variants were not associated with these variables in this sample. 


\subsection{Effect of SLCO1B1 and SLCO2B1 Polymorphisms on Atorvastatin Response}

Results from one-way ANOVA regarding the effect of SLCO1B1 and SLCO2B1 SNPs on total and LDL cholesterol are presented in Table 2. For SLCO1B1 c.388A $>$ G polymorphism, homozygous for c.388G allele presented higher mean percentage of LDL cholesterol reduction than carriers of c.388A allele $(41.3 \pm 12.4 \%$ for GG vs. $36.6 \pm 12.1 \%$ for AA + AG, $p=0.034)$, in a dominant model. For $S L C O 2 B 1$ polymorphism there was no association between lipid parameters and the genotypes.

Table 2. Association of $S L C O 1 B 1$ and $S L C O 2 B 1$ variants with total and LDL cholesterol in individuals treated with atorvastatin.

\begin{tabular}{|c|c|c|c|c|c|c|}
\hline \multirow{2}{*}{ SNP } & \multicolumn{2}{|c|}{ Basal } & \multicolumn{2}{|c|}{ Atorvastatin } & \multicolumn{2}{|c|}{ Change (\%) } \\
\hline & TC & LDL-C & TC & LDL-C & TC & LDL-C \\
\hline \multicolumn{7}{|l|}{ SLCO1B1 } \\
\hline \multicolumn{7}{|l|}{ c.521T $>C$} \\
\hline TT (108) & $281 \pm 37$ & $192 \pm 34$ & $199 \pm 29$ & $118 \pm 26$ & $28.7 \pm 9.1$ & $38.1 \pm 12.4$ \\
\hline $\mathrm{TC}+\mathrm{CC}(28)$ & $282 \pm 35$ & $193 \pm 31$ & $192 \pm 32$ & $114 \pm 28$ & $31.8 \pm 9.3$ & $40.9 \pm 11.6$ \\
\hline$P$ & 0.890 & 0.942 & 0.253 & 0.442 & 0.171 & 0.433 \\
\hline \multicolumn{7}{|l|}{ c. $388 \mathrm{~A}>\mathrm{G}$} \\
\hline GG (49) & $279 \pm 32$ & $193 \pm 36$ & $193 \pm 29$ & $111 \pm 25$ & $30.6 \pm 9.8$ & $41.3 \pm 12.4$ \\
\hline $\mathrm{AA}+\mathrm{AG}(82)$ & $280 \pm 40$ & $191 \pm 28$ & $200 \pm 31$ & $121 \pm 27$ & $28.0 \pm 9.2$ & $36.6 \pm 12.1$ \\
\hline$P$ & 0.550 & 0.527 & 0.162 & 0.077 & 0.123 & 0.034 \\
\hline \multicolumn{7}{|l|}{ c. $463 C>A$} \\
\hline CC (95) & $283 \pm 38$ & $196 \pm 35$ & $199 \pm 32$ & $120 \pm 27$ & $29.4 \pm 9.3$ & $38.4 \pm 11.5$ \\
\hline $\mathrm{CA}+\mathrm{AA}(41)$ & $271 \pm 31$ & $184 \pm 27$ & $194 \pm 27$ & $113 \pm 26$ & $27.9 \pm 9.9$ & $38.0 \pm 14.4$ \\
\hline$P$ & 0.070 & 0.072 & 0.374 & 0.198 & 0.414 & 0.871 \\
\hline \multicolumn{7}{|l|}{ SLCO2B1 } \\
\hline \multicolumn{7}{|l|}{$-71 \mathrm{~T}>\mathrm{C}$} \\
\hline TT (42) & $281 \pm 43$ & $194 \pm 37$ & $200 \pm 34$ & $120 \pm 28$ & $28.6 \pm 8.6$ & $37.6 \pm 10.6$ \\
\hline $\mathrm{TC}+\mathrm{CC}(94)$ & $282 \pm 35$ & $198 \pm 29$ & $198 \pm 29$ & $116 \pm 27$ & $29.4 \pm 9.4$ & $39.0 \pm 12.9$ \\
\hline$P$ & 0.891 & 0.794 & 0.598 & 0.324 & 0.463 & 0.394 \\
\hline
\end{tabular}

In addition, the effect of SLCO1B1 haplotypes on total and LDL cholesterol before and after atorvastatin treatment was investigated. We have compared the effect of *15 homozygous $(* 15 / * 15)$, $* 15$ heterozygous $(* 1 \mathrm{a} / * 15$ and $* 1 \mathrm{~b} / * 15)$ and $* 15$ non-carriers. $(* 1 \mathrm{a}, * 1 \mathrm{~b}$, and $* 1 \mathrm{a} / * 1 \mathrm{~b})$. Despite the fact that $* 15 / * 15$ subjects presented lower total and LDL cholesterol reductions than $* 15$ heterozygous and $* 15$ non-carriers, this association lacked statistical significance (Figure 1). There was no effect of *14 allele on atorvastatin response.

After atorvastatin treatment, LDL cholesterol serum concentrations varied largely from reduction of $61.7 \%$ to $6.4 \%$. Therefore, individuals with LDL cholesterol in the first quartile (reduction higher than $48 \%$ ) were compared with those with lower response. First, a stepwise forward multiple regression analysis including all parameters (age, BMI, gender, basal LDL cholesterol, and c.388A $>$ G genotypes) was performed. After this analysis we concluded that BMI and gender were not related to atorvastatin 
response. Then, a multivariate logistic regression including all the remaining parameters was performed. Results from logistic regression showed that SLCO1B1 c.388GG and higher LDL basal levels were the most significant factors positively related to atorvastatin response (Table 3 ).

Figure 1. Influence of the $S L C O 1 B 1 * 15$ variant on reduction of total (TC) and low-density lipoprotein (LDL-C) cholesterol in response to atorvastatin (10 mg/day/4 weeks). $P>0.05$, as compared by One-Way Analysis of Variance followed by Hom-Sidak test. Number of individuals in parenthesis.

(70)

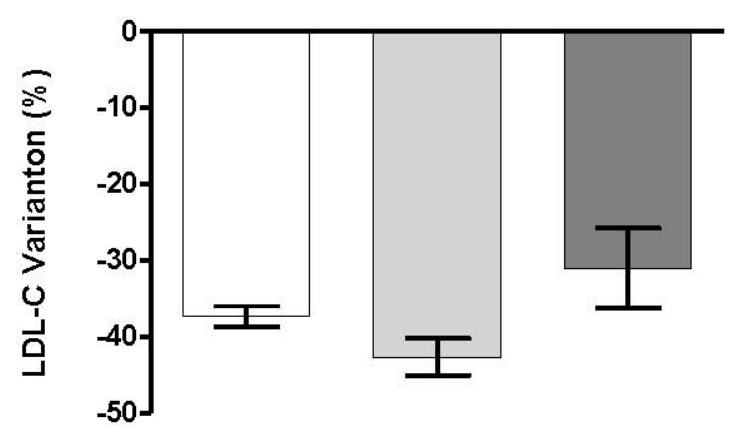

(70)

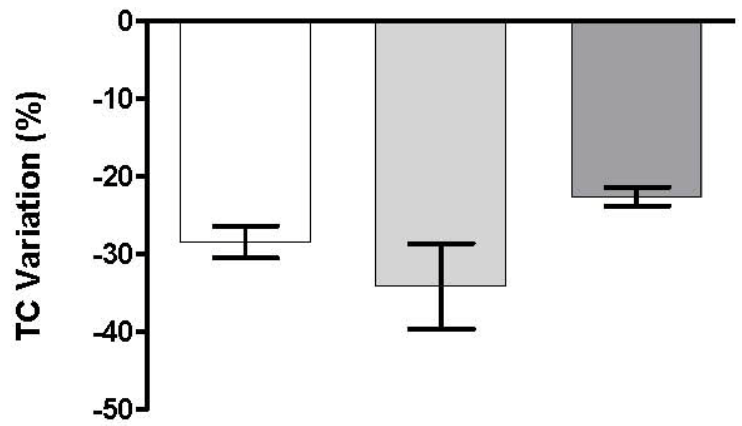

$\square * 1 a /^{*} 1 a+* 1 b / * 1 b+* 1 a /^{*} 1 b$

$\square * 1 a{ }^{*} 15+* 1 b / * 15$ $* 15 / * 15$

Table 3. Multiple logistic regression analysis for reduction of LDL cholesterol after atorvastatin treatment.

\begin{tabular}{cccc}
\hline Variables & $\boldsymbol{p}$-value & Odds Ratio & $\mathbf{9 5 \%}$ CI \\
\hline Basal LDL cholesterol $(\geq 208 \mathrm{mg} / \mathrm{dL})$ & $\mathbf{0 . 0 1 2}$ & $\mathbf{3 . 4 7}$ & $\mathbf{1 . 3 2 - 9 . 1 4}$ \\
Age $(<60$ years $)$ & 0.077 & 0.45 & $0.19-1.09$ \\
$S L C O 1 B 1$ c.388G allele (dominant) & $\mathbf{0 . 0 1 2}$ & $\mathbf{3 . 2 3}$ & $\mathbf{1 . 3 0 - 8 . 0 4}$ \\
\hline
\end{tabular}

CI: Confidence interval; Significant values are highlighted in bold. LDL cholesterol reduction was considered higher than $48 \%$ of the basal level.

SLCO1B1 and SLCO2B1 polymorphisms may have particularly important consequences for cholesterol-lowering therapy with HMGCR inhibitors, as OATPs (1A2, 1B1, 1B3, and 2B1) are involved in the hepatic uptake of statins [5]. Current knowledge has shown that SNPs in SLCO1B1 may result in reduced efficacy and increased risk of systemic exposure, leading to adverse effects [5].

Studies of SLCO1B1 SNPs have focused mainly on c.521T $>$ C polymorphism. They have shown that c.521C allele causes reduced OATP1B1 activity, thus increasing plasma concentrations of all statins except fluvastatin [6-12]. The area under the curve (AUC) of atorvastatin was 1.5-2.0-fold higher in subjects with the 521C/C genotype than in those with the 521T/T [6,7,9].

The effect of c.521T $>\mathrm{C}$ polymorphism on atorvastatin therapy has been investigated in this study. We have found no association between c.521C allele carriers and changes in lipid parameters after 4 weeks of atorvastatin treatment. One reason for that lack of association may be due to a limited number of subjects with $521 \mathrm{C} / \mathrm{C}$ genotype. Because only two individuals in our sample were homozygous for the variant allele they were pooled with the 521C/T genotype, then we could not effectively analyze the effect of $521 \mathrm{C} / \mathrm{C}$ genotype. 
Some studies characterizing the impact of SLCO1B1 polymorphisms on lipid-lowering response have been conducted, however they mainly target pravastatin therapy [15,26-30]. Because these studies were very heterogeneous among the study population (healthy, hypercholesterolemic or elderly subjects), duration of treatment (single dose, 3 or 8 weeks, 1 year) and daily dose (20, 40 or $9.4 \mathrm{mg}$ /day), divergent findings have been reported. For instance, Zhang et al. (2007) [30] reported an attenuated pravastatin ( $20 \mathrm{mg}$ /day for 30 days) pharmacodynamic effect on total cholesterol in patients with 521TC heterozygous compared to 521TT homozygous. On the other hand, treatment with $40 \mathrm{mg}$ pravastatin for 3 weeks caused no difference in lipid-lowering efficacy between c.521C carriers (i.e., SLCO1B1*15 and *17) and non-carriers (SLCO1B1*1a).

The SLCO1B1 c.463C $>$ A polymorphism has been previously associated with fluvastatin response [16]. Carriers of $* 14$ allele had better response to fluvastatin as compared to $* 1 \mathrm{a} / * 1 \mathrm{a}$ or $* 1 \mathrm{a} /{ }^{*} 14$ genotypes. We have found no association between c.463C $>\mathrm{A}$ variant and atorvastatin response. In fact, this is not the first study to describe a lack of association between c.463C $>$ A SNP and atorvastatin response. Thompson et al. (2005) [31] using a much larger sample $(n=1902)$ also did not find any association between this polymorphism and response to atorvastatin. The lack of effect of this polymorphism on atorvastatin response may be due to a substrate-specific effect of this OATP1B1 variant. This substrate-specific effect has been clearly shown for SLCO1B1 c.521T>C SNP. It has been associated with a markedly reduced uptake of all statins except fluvastatin, as discussed before. Then, it is possible that $S L C O 1 B 1 \mathrm{c} .463 \mathrm{C}>\mathrm{A}$ variant has a high affinity for fluvastatin, however it needs to be verified by transporter function analyses.

Significantly high reduction of LDL cholesterol in response to atorvastatin treatment was found in individuals homozygous for SLCO1B1 c.388G allele when compared to c.388A allele carriers $(-41.3$ vs. $-36.6 \%)$. This finding is consistent with previous in vivo studies reporting a higher transport function for OATP1B1 in subjects carrying * $1 b$ variant, resulting in lower oral bioavailability of pravastatin [8] and pitavastatin [32].

There is some evidence that $S L C O 1 B 1 * 15$ variant (c.388G and c.521C) exhibits reduced transport function and play an important role in pravastatin and atorvastatin systemic exposure and elimination [6,33-35]. Lee et al. (2010) [35] have shown that the AUC of atorvastatin was 1.8 higher in $* 15 / * 15$ subjects than in $1 \mathrm{a} / * 15$ and $* 1 \mathrm{~b} / * 15$ and 2.2 -fold than for $* 1 \mathrm{a} / * 1 \mathrm{a}, * 1 \mathrm{a} / * 1 \mathrm{~b}$ and $* 1 \mathrm{~b} / *^{*} 1 \mathrm{~b}$. Haplotype analysis revealed that mean percentage reduction in total and LDL cholesterol values at 4 weeks post-treatment with atorvastatin were lower in $* 15 / * 15$ than in $* 15$ heterozygous and

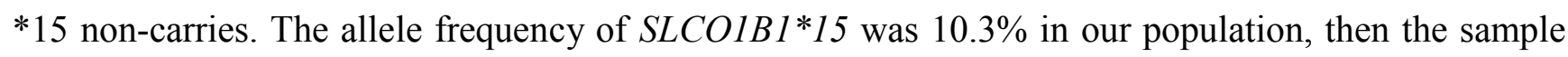
size was not enough to find many subjects carrying *15/*15 genotype, so the association lacks statistical significance. Multiple regression analysis in the study population revealed that only c.388GG was correlated with statin response.

With respect to $S L C O 2 B 1$ polymorphism we have not found significant differences between the different genotypes and atorvastatin response. A previous study also failed to find relationship between polymorphisms of $S L C O 2 B 1$ and pharmacokinetics of pravastatin [7]. 


\section{Material and Methods}

\subsection{Subjects and Study Protocol}

The characteristics of study design have been previously reported [18]. Briefly, 136 hypercholesterolemic (HC) individuals were selected randomly among the outpatients evaluated for the presence of risk factors for coronary artery disease (CAD) at the University Hospital of the Sao Paulo University (Sao Paulo City, Brazil). The study protocol was approved by the Ethics Committee of this institution as well the Committee of the Faculty of Pharmaceutical Sciences (University of Sao Paulo). Individuals diagnosed with thyroid, liver and kidney diseases, diabetes, and triglycerides higher than $400 \mathrm{mg} / \mathrm{dL}$ or subjects under treatment with lipid-lowering drugs, hormone replacement or oral contraceptives were not included. Pregnant women or patients with heart disease known previously were not included too.

Information on age, body mass index (BMI), gender, hypertension, obesity, menopause status, cigarette smoking, physical activity, alcohol consumption and family history of CAD were recorded.

HC patients with (LDL) cholesterol higher than $160 \mathrm{mg} / \mathrm{dL}$, even after a low cholesterol diet during 4 weeks, were started on atorvastatin therapy, $10 \mathrm{mg}$ orally once daily for 4 weeks. At the end of the protocol, the patients had a last appointment with the doctor and response to atorvastatin as well as any possible adverse reactions was evaluated. The study design was based on the recommendations of the National Cholesterol Education Program (NCEP) for treatment of high blood cholesterol in adults [36]. The dose of $10 \mathrm{mg}$ atorvastatin was chosen because the patients had moderate elevations of LDL cholesterol, and LDL cholesterol goal will be achieved with low doses for these patients. In addition, the NCEP recommend checking the response to drug therapy in about 6 weeks.

Response to atorvastatin was evaluated by reduction of LDL cholesterol after the treatment, and adverse effects were monitored by measuring creatine kinase (CK) and alanine aminotransferase (ALT) enzymes.

\subsection{Biochemical Profile and SLCO Variants Genotyping}

Blood samples for biochemical profile (lipids, CK, and ALT) measurements and genomic DNA extraction were collected after an overnight fast, one day before and 4 weeks after atorvastatin treatment. All patients followed exactly the same study protocol. Laboratory methods for biochemical parameters are described elsewhere [18].

Genomic DNA was extracted from EDTA-anticoagulated blood by a salting-out procedure optimized in our laboratory [37]. Polymorphisms of SLCO1B1 [c.521T >C (Val174Ala, rs4149056), c.388A $>$ G (Asp130Asn, rs2306283), c.463C $>$ A (Pro155Thr, rs11045819)], and SLCO2B1 [-71T >C (rs2851069)] were detected by TaqMan ${ }^{\circledR}$ Real time PCR. TaqMan Drug Metabolism Genotyping Assay (20X) were obtained from Life Technologies (Foster City, CA, USA).

PCR assays contained $4 \mu \mathrm{L}$ of Universal Master Mix (2X) (Life Technologies), $0.4 \mu \mathrm{L}$ of TaqMan Drug Metabolism Genotyping Assay (20X) and $3.6 \mu \mathrm{L}$ de DNA (20 ng) diluted in nuclease-free water. The thermal cycling protocol consisted of initial cycle at $10 \mathrm{~min}$ a $95{ }^{\circ} \mathrm{C}$ followed by 40 cycles at $92{ }^{\circ} \mathrm{C}$ for $15 \mathrm{~s}, 60{ }^{\circ} \mathrm{C}$ for $1 \mathrm{~min}$, using standard 7500 conditions. For SLCO2B1 polymorphisms the cycles were increased to 50 , and the time for extension was $90 \mathrm{~s}$. The amplification was carried out in a 
7500 fast real-time system (Life Technologies). Genotype calling was performed using the SDS software (Life Technologies).

\subsection{Ancestry Informative Markers (AIMs)}

The ancestral origin and African component ancestry (ACA) of the individuals was explored using a 34-plex AIM-SNPs assay. SNPs were genotyped by multiplex-PCR followed by 34-plex SNaPshot ${ }^{\circledR}$ primer extension reactions (Life Technologies, Foster City, USA). Extension products were separated by capillary electrophoresis (3130 Analyzer, Life Technologies) and POP6 ${ }^{\mathrm{TM}}$ polymer (details in [38]). The ACA of the samples was estimated and categorized into four ancestral categories $(<0.25$; $0.25-0.50 ; 0.50-0.75 ;>0.75)$ according to the relative contribution of a variable number of African ancestral population.

\subsection{Statistical Analysis}

For SLCO1B1, as previously described by Tirona and colleagues (2002) [39], haplotypes were defined based on the presence of c.388A $>\mathrm{G}, \mathrm{c} .463 \mathrm{C}>\mathrm{A}$ and c.521T $>\mathrm{C}$ polymorphisms alone or in combination, as follows: SLCO1B1*la (wild type), *1b (c.388G), *4 (c.463A), *5 (c.521C), $* 14$ (c.388G and c.463A) or *15 (c.388G and c.521C). The agreement of genotypes frequencies with Hardy-Weinberg equilibrium expectations was tested by $\chi^{2}$ test using Haploview software. Relationships between the genotypes or haplotypes and categorical variables were evaluated by the Chi-square or Exact Fisher test.

Continuous variables are presented as mean $\pm \mathrm{SD}$. Those without normal distribution were analyzed by a non parametric test, and they are presented as median $(25 \%-75 \%)$. Numerical variables were compared by $\mathrm{t}$ test (two variables) and One-way ANOVA (three or more variables) and Holm-Sidak method was used for multiple comparisons. Logistic regression analysis was used to evaluate the relationships between reduction of serum LDL cholesterol and other variables after treatment with atorvastatin. Statistical tests were performed using the Sigma Stat version 3.5 (SPSS Inc., Chicago, IL, USA). Significance was considered $P<0.05$.

\section{Conclusions}

The lack of association between lipid response to atorvastatin and SLCO1B1 c.521T >C polymorphism may be due to the size of our sample since we could not find many individuals homozygous to the rare allele. This caused the statistical power of the test performed to be below the desired level. In addition, the positive association between c.388GG carriers and higher LDL cholesterol reductions would be greatly strengthened if the sample were larger.

SLCO1B1 c.388A $>$ G polymorphism causes significant increase in atorvastatin response and may be an important marker for predicting efficacy of lipid-lowering therapy. However, others factors, such as the drug given to the patient, duration of the treatment, daily dose, basal LDL cholesterol, may influence the efficacy of the therapy and needs to be taken into consideration. 


\section{Acknowledgments}

This work was supported by grants from FAPESP (2008/06667-9). A.C. Rodrigues, F.D.V. Genvigir and M.A.V. Willrich are recipients of fellowships from FAPESP, Sao Paulo, Brazil. M.H. Hirata and R.D.C. Hirata are recipients of fellowships from CNPq, Brasilia, Brazil.

\section{Disclosure}

This work was partially supported by Life Technologies, Sao Paulo, SP, Brazil. The sponsor of this study had no role in the study design, data collection, data analysis, data interpretation or writing of the report. S.G. Purim was salaried personnel of Life Technologies. The authors have no other relevant affiliation or financial involvement with any organization or entity with a financial interest in or financial conflict with the subject matter or materials in the manuscript apart from those disclosed.

\section{References}

1. Ieri, I.; Higuchi, S.; Sugiyama, Y. Genetic polymorphisms of uptake (OATP1B1, 1B3) and efflux (MRP2, BCRP) transporters: Implications for inter-individual differences in the pharmacokinetics and pharmacodynamics of statins and other clinically relevant drugs. Expert Opin. Drug Metab. Toxicol. 2009, 5, 703-729.

2. Nawrocki, J.W.; Weiss, S.R.; Davidson, M.H.; Sprecher, D.L.; Schwartz, S.L.; Lupien, P.J.; Jones, P.H.; Haber, H.E.; Black, D.M. Reduction of LDL cholesterol by $25 \%$ to $60 \%$ in patients with primary hypercholesterolemia by atorvastatin, a new HMG-CoA reductase inhibitor. Arterioscler. Thromb. Vasc. Biol. 1995, 15, 678-682.

3. Vaughan, C.J.; Gotto, A.M., Jr. Update on statins: 2003. Circulation 2004, 110, 886-892.

4. Shitara, Y.; Sugiyama, Y. Pharmacokinetic and pharmacodynamic alterations of 3-hydroxy-3methylglutaryl coenzyme A (HMG-CoA) reductase inhibitors: Drug-drug interactions and interindividual differences in transporter and metabolic enzyme functions. Pharmacol. Ther. 2006, 112, 71-105.

5. Rodrigues, A.C. Efflux and uptake transporters as determinants of statin response. Expert Opin. Drug Metab. Toxicol. 2010, 6, 621-632.

6. Nishizato, Y.; Ieiri, I.; Suzuki, H.; Kimura, M.; Kawabata, K.; Hirota, T.; Takane, H.; Irie, S.; Kusuhara, H.; Urasaki, Y.; Urae, A.; Higuchi, S.; Otsubo, K.; Sugiyama, Y. Polymorphisms of OATP-C (SLC21A6) and OAT3 (SLC22A8) genes: Consequences for pravastatin pharmacokinetics. Clin Pharmacol. Ther. 2003, 73, 554-565.

7. Niemi, M.; Schaeffeler, E.; Lang, T.; Fromm, M.F.; Neuvonen, M.; Kyrklund, C.; Backman, J.T.; Kerb, R.; Schwab, M.; Neuvonen, P.J.; Eichelbaum, M.; Kivistö, K.T. High plasma pravastatin concentrations are associated with single nucleotide polymorphisms and haplotypes of organic transporting polypeptide-C (OATP-C, SLCO1B1). Pharmacogenetics 2004, 14, 429-440.

8. Chung, J.Y.; Cho, J.Y.; Yu, K.S.; Kim, J.R.; Oh, D.S.; Jung, H.R.; Lim, K.S.; Moon, K.H.; Shin, S.G.; Jang, I.J. Effect of OATP1B1 (SLCO1B1) variants on the pharmacokinetics of pitavastatin in healthy volunteers. Clin. Pharmacol. Ther. 2005, 78, 342-350. 
9. Niemi, M.; Pasanen, M.K.; Neuvonen, P.J. SLCO1B1 polymorphisms and sex affect the pharmacokinetics of pravastatin but not fluvastatin. Clin. Pharmacol. Ther. 2006, 80, 356-366.

10. Pasanen, M.K.; Fredrikson, H.; Neuvonen, P.J.; Niemi, M. Different effects of SLCO1B1 polymorphisms on the pharmacokinetics of atorvastatin and rosuvastatin. Clin. Pharmacol. Ther. 2007, 82, 726-733.

11. Ieiri, I.; Suwannakul, S.; Maeda, K.; Uchimaru, H.; Hashimoto, K.; Kimura, M.; Fujino, H.; Hirano, M.; Kusuhara, H.; Irie, S.; Higuchi, S.; Sugiyama, Y. SLCO1B1 (OATP1B1, an uptake transporter) and ABCG2 (BCRP, an efflux transporter) variant alleles and pharmacokinetics of pitavastatin in healthy volunteers. Clin. Pharmacol. Ther. 2007, 82, 541-547.

12. Choi, J.H.; Lee, M.G.; Cho, J.Y.; Lee, J.E.; Kim, K.H.; Park, K. Influence of OATP1B1 genotype on the pharmacokinetics of rosuvastatin in Koreans. Clin. Pharmacol. Ther. 2008 83, 251-257.

13. Link, E.; Parish, S.; Armitage, J.; Bowman, L.; Heath, S.; Matsuda, F.; Gut, I.; Lathrop, M.; Collins, R. SLCO1B1 variants and statin-induced myopathy-A genomewide study. N. Engl. J. Med. 2008, 359, 789-799.

14. Mwinyi, J.; Johne, A.; Bauer, S.; Roots, I.; Gerloff, T. Evidence for inverse effects of OATP-C (SLC21A6) *5 and *1b haplotypes on pravastatin kinetics. Clin. Pharmacol. Ther. 2004, 75, 415-421.

15. Takane, H.; Miyata, M.; Burioka, N.; Shigemasa, C.; Shimizu, E.; Otsubo, K.; Ieiri, I. Pharmacogenetic determinants of variability in lipid-lowering response to pravastatin therapy. J. Hum. Gene. 2006, 51, 822-826.

16. Couvert, P.; Giral, P.; Dejager, S.; Gu, J.; Huby, T.; Chapman, M.J.; Bruckert, E.; Carrié, A. Association between a frequent allele of the gene encoding OATP1B1 and enhanced LDL-lowering response to fluvastatin therapy. Pharmacogenomics 2008, 9, 1217-1227.

17. Knauer, M.J.; Urquhart, B.L.; Meyer zu Schwabedissen, H.E.; Schwarz, U.I.; Lemke, C.J.; Leake, B.F.; Kim, R.B.; Tirona, R.G. Human skeletal muscle drug transporters determine local exposure and toxicity of statins. Circ. Res. 2010, 106, 297-306.

18. Rebecchi, I.M.; Rodrigues, A.C.; Arazi, S.S.; Genvigir, F.D.; Willrich, M.A.; Hirata, M.H.; Soares, S.A.; Bertolami, M.C.; Faludi, A.A.; Bernik, M.M.; Dorea, E.L.; Dagli, M.L.; Avanzo, J.L.; Hirata, R.D. ABCB1 and ABCC1 expression in peripheral mononuclear cells is influenced by gene polymorphisms and atorvastatin treatment. Biochem. Pharmacol. 2009, 77, $66-75$.

19. Pasanen, M.K.; Neuvonen, P.J.; Niemi, M. Global analysis of genetic variation in SLCO1B1. Pharmacogenomics 2008, 9, 19-33.

20. Mwinyi, J.; Köpke, K.; Schaefer, M.; Roots, I.; Gerloff, T. Comparison of SLCO1B1 sequence variability among German, Turkish, and African populations. Eur. J. Clin. Pharmacol. 2008, 64, 257-266.

21. Xu, L.Y.; He, Y.J.; Zhang, W.; Deng, S.; Li, Q.; Zhang, W.X.; Liu, Z.Q.; Wang, D.; Huang, Y.F.; Zhou, H.H.; Sun, Z.Q. Organic anion transporting polypeptide-1B1 haplotypes in Chinese patients. Acta Pharmacol. Sin. 2007, 28, 1693-1697.

22. Pena, S.D.; Bastos-Rodrigues, L.; Pimenta, J.R.; Bydlowski, S.P. DNA tests probe the genomic ancestry of Brazilians. Braz. J. Med. Biol. Res. 2009, 42, 870-876. 
23. Suarez-Kurtz, G.; Perini, J.A.; Bastos-Rodrigues, L.; Pena, S.D.; Struchiner, C. Impact of population admixture on the distribution of the CYP3A5*3 polymorphism. Pharmacogenomics 2007, 8, 1299-1306.

24. Estrela, R.C.; Ribeiro, F.S.; Carvalho, R.S.; Gregório, S.P.; Dias-Neto, E.; Struchiner, C.J.; Suarez-Kurtz, G. Distribution of ABCB1 polymorphisms among Brazilians: Impact of population admixture. Pharmacogenomics 2008, 9, 267-276.

25. Suarez-Kurtz, G.; Amorim, A.; Damasceno, A.; Hutz, M.H.; de Moraes, M.O.; Ojopi, E.B.; Pena, S.D.; Perini, J.A.; Prata, M.J.; Ribeiro-dos-Santos, A.; Romano-Silva, M.A.; Teixeira, D.; Struchiner, C.J. VKORC1 polymorphisms in Brazilians: Comparison with the Portuguese and Portuguese-speaking Africans and pharmacogenetic implications. Pharmacogenomics 2010, 11, 1257-1267.

26. Niemi, M.; Neuvonen, P.J.; Hofmann, U.; Backman, J.T.; Schwab, M.; Lütjohann, D.; von Bergmann, K.; Eichelbaum, M.; Kivistö, K.T. Acute effects of pravastatin on cholesterol synthesis are associated with SLCO1B1 (encoding OATP1B1) haplotype *17. Pharmacogenet. Genomics 2005, 15, 303-309.

27. Igel, M.; Arnold, K.A.; Niemi, M.; Hofmann, U.; Schwab, M.; Lütjohann, D.; von Bergmann, K.; Eichelbaum, M.; Kivistö, K.T. Impact of the SLCO1B1 polymorphism on the pharmacokinetics and lipid-lowering efficacy of multiple-dose pravastatin. Clin. Pharmacol. Ther. 2006, 79, $419-426$.

28. Gerloff, T.; Schaefer, M.; Mwinyi, J.; Johne, A.; Sudhop, T.; Lütjohann, D.; Roots, I.; von Bergmann, K. Influence of the SLCO1B1*1b and *5 haplotypes on pravastatin's cholesterol lowering capabilities and basal sterol serum levels. Naunyn Schmiedebergs Arch. Pharmacol. 2006, 373, 45-50.

29. Hedman, M.; Antikainen, M.; Holmberg, C.; Neuvonen, M.; Eichelbaum, M.; Kivistö, K.T.; Neuvonen, P.J.; Niemi, M. Pharmacokinetics and response to pravastatin in paediatric patients with familial hypercholesterolaemia and in paediatric cardiac transplant recipients in relation to polymorphisms of the SLCO1B1 and ABCB1 genes. Br. J. Clin. Pharmacol. 2006, 61, 706-715.

30. Zhang, W.; Chen, B.L.; Ozdemir, V.; He, Y.J.; Zhou, G.; Peng, D.D.; Deng, S.; Xie, Q.Y.; Xie, W.; Xu, L.Y.; Wang, L.C.; Fan, L.; Wang, A.; Zhou, H.H. SLCO1B1 521>T functional genetic polymorphism and lipid-lowering efficacy of multiple-dose pravastatin, in Chinese coronary heart disease patients. Br. J. Clin. Pharmacol. 2007, 64, 346-352.

31. Thompson, J.F.; Man, M.; Johnson, K.J.; Wood, L.S.; Lira, M.E.; Lloyd, D.B.; Banerjee, P.; Milos, P.M.; Myrand, S.P.; Paulauskis, J.; Milad, M.A.; Sasiela, W.J. An association study of 43 SNPs in 16 candidate genes with atorvastatin response. Pharmacogenomics J. 2005, 5, 352-358.

32. Wen, J.; Xiong, Y. OATP1B1 388A $>$ G polymorphism and pharmacokinetics of pitavastatin in Chinese healthy volunteers. J. Clin. Pharm. Ther. 2010, 35, 99-104.

33. Iwai, M.; Suzuki, H.; Ieiri, I.; Otsubo, K.; Sugiyama, Y. Functional analysis of single nucleotide polymorphisms of hepatic organic anion transporter OATP1B1 (OATP-C). Pharmacogenetics 2004, 14, 749-757. 
34. Kameyama, Y.; Yamashita, K.; Kobayashi, K.; Hosokawa, M.; Chiba, K. Functional characterization of SLCO1B1 (OATP-C) variants, SLCO1B1*5, SLCO1B1*15 and SLCO1B1*15+C1007G, by using transient expression systems of HeLa and HEK293 cells. Pharmacogenet. Genomics 2005, 15, 513-522.

35. Lee, Y.J.; Lee, M.G.; Lim, L.A.; Jang, S.B.; Chung, J.Y. Effects of SLCO1B1 and ABCB1 genotypes on the pharmacokinetics of atorvastatin and 2-hydroxyatorvastatin in healthy Korean subjects. Int. J. Clin. Pharmacol. Ther. 2010, 48, 36-45.

36. National Cholesterol Education Program (NCEP). Third Report of the Expert Panel on Detection, Evaluation, and Treatment of High Blood Cholesterol in Adults (Adult Treatment Panel III), Final report. Circulation 2002, 106, 3143-3421.

37. Salazar, L.A.; Hirata, M.H.; Cavalli, S.A.; Machado, M.O.; Hirata, R.D. Optimized procedure for DNA isolation from fresh and cryopreserved clotted human blood useful in clinical molecular testing. Clin. Chem. 1998, 44, 1748-1750.

38. Phillips, C.; Salas, A.; Sánchez, J.J.; Fondevila, M.; Gómez-Tato, A.; Alvarez-Dios, J.; Calaza, M.; de Cal, M.C.; Ballard, D.; Lareu, M.V.; Carracedo, A. SNPforID Consortium. Inferring ancestral origin using a single multiplex assay of ancestry-informative marker SNPs. Forensic Sci. Int. Genet. 2007, 1, 273-280.

39. Tirona, R.G.; Leake, B.F.; Merino, G.; Kim, R.B. Polymorphisms in OATP-C: Identification of multiple allelic variants associated with altered transport activity among European- and African-Americans. J. Biol. Chem. 2001, 276, 35669-35675.

(C) 2011 by the authors; licensee MDPI, Basel, Switzerland. This article is an open access article distributed under the terms and conditions of the Creative Commons Attribution license (http://creativecommons.org/licenses/by/3.0/). 